\title{
Modeling the dust and gas temperatures near young stars
}

\author{
Andrea Urban ${ }^{1}$ and Neal J. Evans $\mathrm{II}^{1}$ \\ ${ }^{1}$ Department of Astronomy, University of Texas at Austin, Austin, TX 78712, USA \\ email: aurban@astro.as.utexas.edu.
}

\begin{abstract}
As young stars form, they interact with their environment in many ways. We study the radiative interaction of a young star with its surrounding cluster environment. The change in gas temperature caused by a forming star can trigger the formation or inhibit the growth of nearby star forming cores. We calculate the gas temperature around a single star by balancing the dust-gas collisional heating, molecular cooling, and cosmic ray heating rates for a grid of models with various luminosities and density distributions. In the future, this work can be used in large-scale simulations of clustered star formation to study the effect of using a gas temperature which depends not only on density, but also on radiative environment.
\end{abstract}

Keywords. stars: formation, methods: numerical

\section{Dust and Gas Temperature}

In order to calculate the dust temperature, we have created a grid of models using the spherical radiative transfer code, DUSTY (Ivezic et al. 1999). We assume that the central star is a black body with $\mathrm{T}=10,000 \mathrm{~K}$ and a luminosity between $10^{-2} \mathrm{~L}_{\odot}$ and $10^{5} \mathrm{~L}_{\odot}$. The dust properties are given by OH5 dust (Ossenkopf \& Henning 1994). The spherical density profile is exponentially decreasing. We use an energy rate balance code to calculate the gas temperature (Doty \& Neufeld 1997). We include gas-dust collisional temperature coupling, cosmic-ray heating, and CO cooling (Young et al. 2004). Figure 1 shows an example model.

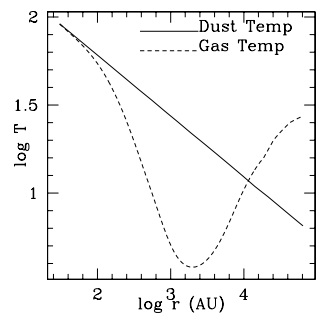

Figure 1. Model Parameters: $\mathrm{L}=10 \mathrm{~L}_{\odot}, \mathrm{n}=10^{4.5} \mathrm{~cm}^{-3}$ at $1000 \mathrm{AU}$. The dust and gas are wellcoupled via collisional heat transfer in the center. As the density decreases, the gas is able to cool efficiently through molecular lines. Cosmic-ray heating becomes important as the density decreases even more.

\section{References}

Doty, S. D. \& Neufeld, D. A. 1997, ApJ 489, 122

Ivezic, Z., Nenkova, M. \& Elitzur, M. 1999, astro-ph/9910475

Ossenkopf, V. \& Henning, T. 1994, A\&A 291, 943

Young, K. E., Lee, J.-E., Evans, N. J., II, Goldsmith, P. F., \& Doty, S. D. 2004, ApJ 614, 252 